\title{
Transition Edge Sensor Fabrication
}

K. L. Nelms, D. Liu, D. McCammon, L. Rocks, W. T. Sanders, P. Tan, J. Vaillancourt

University of Wisconsin-Madison, Department of Physics, 1150 University Ave., Madison, WI, 53706-1390

Improving $\mathrm{x}$-ray spectral energy resolution in conventional scanning electron microscopes (SEM) can be achieved by using microcalorimeters. Although improvements to microcalorimeter design are still being studied, several research groups have developed fabrication techniques that result in consistent devices [1]. Here we discuss the fabrication techniques we use to make microcalorimeters using superconducting transition edge sensors (TES) as thermometers.

We begin by depositing a $1 \mu \mathrm{m}$, low stress silicon nitride layer onto a $<100>$ silicon wafer. A molybdenum (Mo)/copper $(\mathrm{Cu})$ bilayer is then deposited via electron beam evaporation. The Mo layer is $40 \mathrm{~nm}$ thick and is deposited at a substrate temperature of $700{ }^{\circ} \mathrm{C}$. The high substrate temperature helps facilitate optimum grain size and structure of the Mo. Without breaking vacuum, a $180 \mathrm{~nm}$ thick $\mathrm{Cu}$ layer is then deposited at $100^{\circ} \mathrm{C}$.

To obtain our goal of producing a $400 \mu \mathrm{m}$ x $400 \mu \mathrm{m}$ TES pad with Mo leads (see Figure 1) the general micro-processing consists of the following steps:

1. Wet etch two trenches in the $\mathrm{Cu}$ to gain access to the Mo layer. These trenches define the sides of the TES pad.

2. Using the remaining $\mathrm{Cu}$ as a mask, the exposed Mo is etched via dry plasma. This process laterally etches the Mo resulting in the $\mathrm{Cu}$ overhanging the Mo layer by $\sim 300 \mathrm{~nm}$. The $\mathrm{Cu}$ overhang is fabricated to eliminate superconducting shorts along the edges of the device.

3. Wet etch the $\mathrm{Cu}$ to define the ends of the TES pad.

4. Wet etch the Mo to define the leads.

5. The device, to be a good thermometer, must be thermally isolated. We achieve this by etching a window in the silicon substrate. $\mathrm{KOH}$ is used to etch the silicon from the back of the wafer while covering the device to keep it protected. The $\mathrm{KOH}$ etch will follow the (111) planes and stop on the silicon nitride layer.

Two primary problems are present in our fabrication process. One is contamination of the mo/cu interface, which may lead to an unpredictable transition temperature. Two hours and twenty minutes pass between the deposition of the layers due to necessary cooling time of the substrate. During this time, we believe that there is sufficient oxygen content within our electron beam system to contaminate the surface of the moly film. 
The second and biggest challenge we are facing is device repeatability (see Figures 2, 3). To help facilitate consistency between devices, we are investigating improvements to our electron beam system. The installation of an ion gun for surface cleaning and a second mass meter are two of the changes that are being considered.

[1] P. Tan et al., Low Temperature Detectors, (2002) AIP Conference Proceedings No. 605, and references therein.
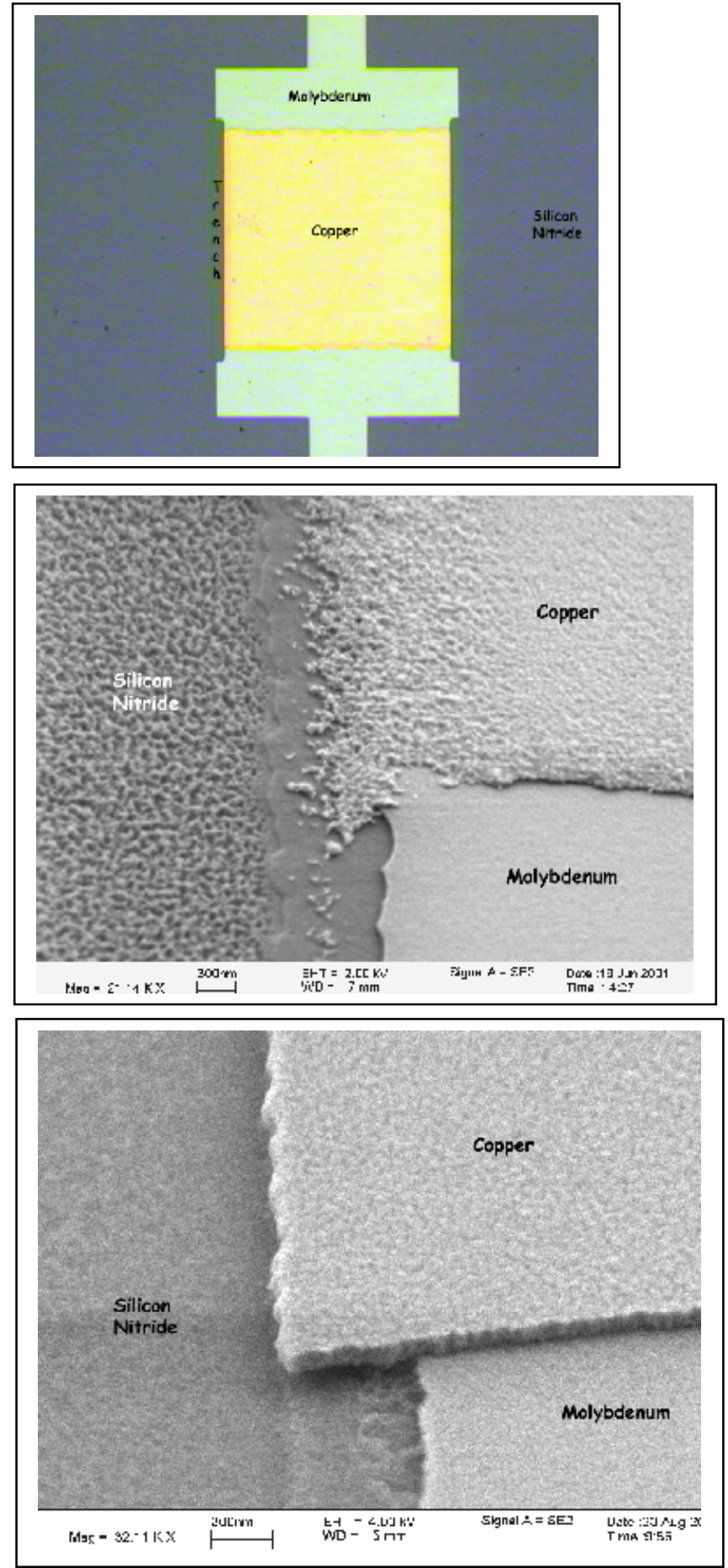

Figure 1: This light microscopy image shows a $400 \mu \mathrm{m}$ x $400 \mu \mathrm{m}$ TES device with Mo leads. The trenches described in step one are visible along the vertical sides of the pad.

Figure 2: This SEM image shows the lower left corner of a bad TES device. Note the loss of $\mathrm{Cu}$ integrity.

Figure 3: This SEM image shows the lower left corner of a good TES device. Note the straight $\mathrm{Cu}$ edge and clean overhang.

**Note: Figures 2 and 3 were devices on the same wafer. This shows the inconsistencies we are observing. 